\title{
Cardiovascular magnetic resonance imaging in hypertrophic cardiomyopathy: Current state of the art
}

\author{
Muhammad Umar Kamal, Irbaz Bin Riaz, Rajesh Janardhanan \\ Sarver Heart Center, Division of Cardiology, Department of Medicine, \\ Banner-University Medical Center, Tucson, United States
}

\begin{abstract}
Hypertrophic cardiomyopathy (HCM) is the most common genetic cardiomyopathy with a prevalence of 1:500 (0.2\%) in the general population. Sudden cardiac death (SCD) is the most feared presentation of HCM. Therefore, it is essential to identify individuals at high risk in order to prevent $S C D$. The absence of conventional risk factors does not nullify the risk of HCM related SCD. Although echocardiography is currently the most widely used imaging modality, cardiac magnetic resonance (CMR) allows detailed characterization of the HCM phenotype, which makes it possible to differentiate HCM from other causes of left ventricular hypertrophy. $C M R$ has the potential to further refine risk stratification. Late gadolinium enhancement (LGE) on CMR is a high-risk feature and there is emerging data to suggest that the presence of $L G E$ should be employed as a marker for major adverse outcomes such as SCD, arrhythmias, systolic and diastolic heart failure. Hence, LGE on CMR may be considered an additional risk factor for SCD in HCM patients and should be incorporated in decision-making for implantable cardioverter defibrillator implantation to aid primary prevention. Novel markers such as the extent of myocardial fibrosis on CMR must be accounted for comprehensive risk stratification of HCM patients. The purpose of this review is to discuss the current status and emerging role of CMR in HCM. (Cardiol J 2016; 23, 3: 250-263)
\end{abstract}

Key words: hypertrophic cardiomyopathy, sudden cardiac death, myocardial fibrosis, cardiovascular magnetic resonance, T1-mapping, gadolinium contrast

\section{Introduction}

Hypertrophic cardiomyopathy (HCM) is the most common genetic cardiomyopathy with a prevalence of $1: 500(0.2 \%)$ in the general population [1]. It is characterized by inappropriate myocardial wall thickening and is usually caused by mutations in genes encoding proteins of the cardiac sarcomere. It is transmitted by an autosomal dominant pattern of inheritance in $60 \%$ of cases. The heterogeneity in penetrance, phenotypic expression, and clinical presentation suggests a role of additional genetic, epigenetic and environmental factors in the pathogenesis. Defective sarcomere proteins impair the function of cardiac myocytes, ultimately resulting in left ventricular hypertrophy (LVH) with asymmetric ventricular septal hypertrophy being the most common phenotype [2, 3]. HCM has a broad range of clinical manifestations of HCM. Most patients remain undiagnosed or asymptomatic with normal life expectancy [4]. However, symptomatic patients present with palpitations, chest

Address for correspondence: Rajesh Janardhanan, MD, MRCP, FACC, FASE, Associate Professor of Medicine and Medical Imaging, Medical Director, Non-Invasive Cardiac Imaging, Banner-University Medical Center South, Sarver Heart Center, Box 245037, 1501 N. Campbell Avenue, Tucson, AZ 85724, USA, tel: 520-626-3766, fax: 520-626-4333, e-mail: raj@shc.arizona.edu

Received: 01.02.2016 Accepted: 08.02.2016 
pain, exertional dyspnea, or sudden cardiac death (SCD). It is the most common cause of SCD in the young and one of the leading causes of heart failure (HF) disability at 5-10 years of age [5]. The symptoms occur due to systolic or diastolic dysfunction, left ventricular (LV) outflow tract obstruction, microvascular ischemia or ventricular/ /supraventricular arrhythmias [6]. Although annual mortality rates range from $1 \%$ to $5 \%$, a significant number of patientsareatrisk for adverse outcomes [7]. Therefore, it is essential to identify the risk of SCD and plan implantable cardioverter defibrillator (ICD) in those patients who are at high risk. Although echocardiography is commonly used, cardiac magnetic resonance (CMR) is considered to be the gold standard for evaluating ventricular mass, volumes, and ejection fraction (EF). Unlike echocardiography, CMR has an advantage in that there are no limitations from poor acoustic windows. The purpose of this review is to discuss the current status and emerging role of CMR in HCM.

\section{Diagnosis of HCM}

Echocardiography has been the most valuable screening tool to evaluate HCM, as it is widely available, bedside technique, which is relatively cheap and non-ionizing. In addition, it provides useful information for risk stratification, treatment, and follow-up of patients.

\section{Cardiac magnetic resonanse for diagnosis of $\mathrm{LVH}$}

Although asymmetrical septal hypertrophy is a common phenotype, accurate assessment of LVH by echocardiography may not be possible in all segments in all patients. It is hard to differentiate the lateral epicardial border of the LV myocardium from the adjacent thoracic parenchyma and the epicardial border of the posterior septum in the area of insertion of the right ventricular (RV) free wall by echocardiography due to loss of spatial resolution. CMR is not limited by such constraints and it is superior to echocardiography for assessing LV wall thickness on the anterior wall, posterior septum and apex of the heart due to its tomographic imaging capability and higher spatial resolution $[3,8]$. Maron et al. [8] described different patterns of LVH in $333 \mathrm{HCM}$ patients utilizing CMR. The most common location of hypertrophy (in $70 \%$ patients) was the confluence of the basal anterior septum with the adjacent anterior free LV wall. The next most common site was the posterior septum at

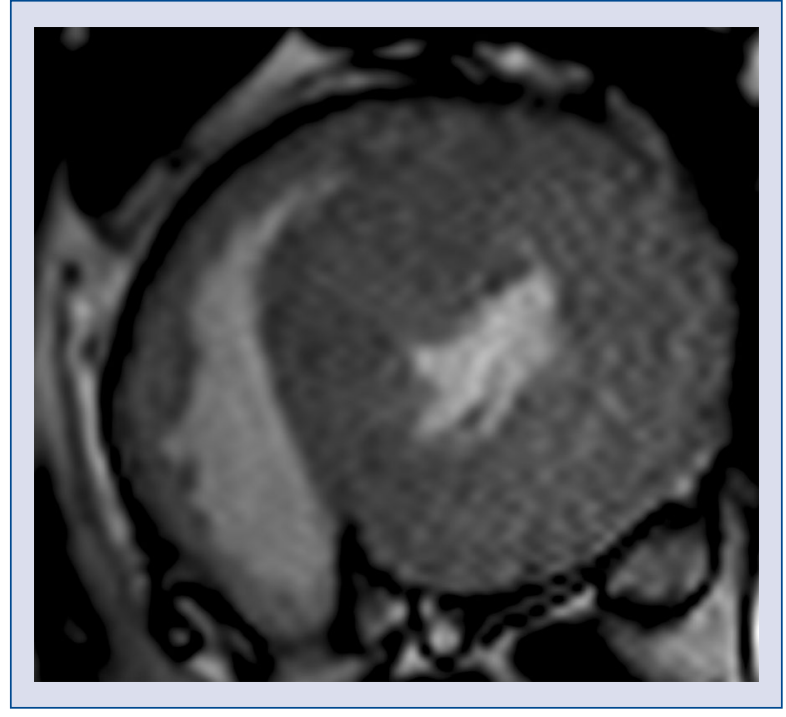

Figure 1. Short axis view on cardiac magnetic resonance demonstrating concentric left ventricular hypertrophy.

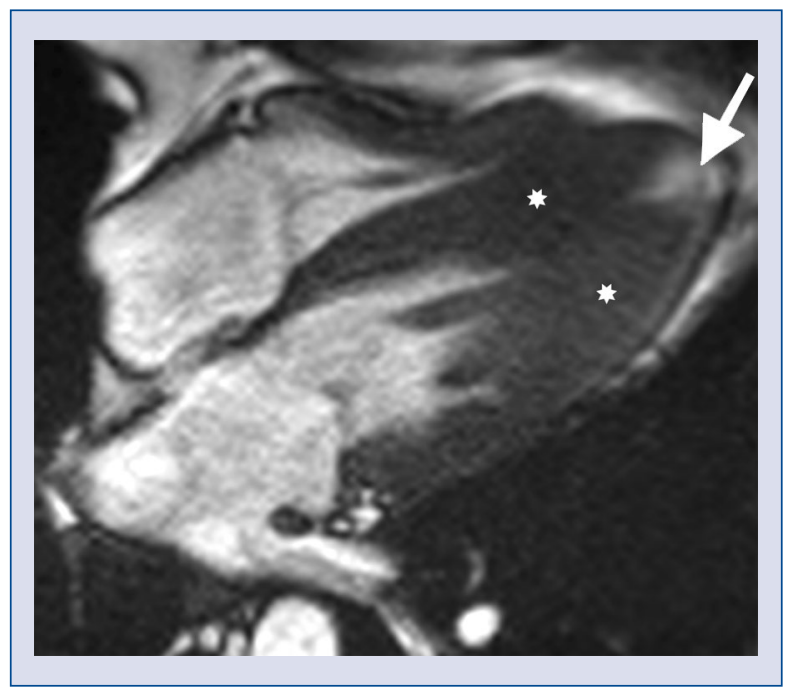

Figure 2. Four chamber view on cardiac magnetic resonance demonstrating mid-ventricular hypertrophy (white asterisk) and an apical aneurysm (white arrow).

the mid-ventricular level [3]. Thus, CMR allows detailed characterization of the HCM phenotype (Figs. 1, 2) and helps differentiate HCM from other causes of LVH.

\section{Differentiating HCM from athlete's heart}

Cardiac magnetic resonanse has several advantages as compared to echocardiography for the evaluation of $\mathrm{LVH}$ in trained athletes. If there is an ambiguity regarding the borderline LV wall thick- 
ness either due to athletic training or HCM, the presence of scar/enhancement on CMR would suggest the presence of HCM. Interruption or cessation of intense physical activities in trained athletes (deconditioning) for short [9] or long periods [10] of time results in restoration of normal LV cavity size due to regression of LVH. However, alteration in physical activity does not affect LV wall thickness in HCM. CMR can be used to detect serial changes in LV wall thickness during deconditioning of the heart in athletes [10].

\section{Detection of myocardial fibrosis with late gadolinium enhancement using CMR}

Myocardial fibrosis is defined as a significant increase in the collagen volume fraction of myocardial tissue, which is common in advanced cardiac disease [11]. Replacement (focal and diffuse) fibrosis or interstitial fibrosis are two types of myocardial fibrosis commonly seen in HCM. Scarring or replacement fibrosis is the accumulation of collagen corresponding to necrotic or damaged myocyte. Interstitial fibrosis is diffusely distributed in the extracellular space. It is predicted that transforming growth factor-beta 1 triggers production of extracellular matrix proteins leading to myocardial fibrosis in HCM [12]. Myocardial fibrosis has been recognized as a predictor of adverse clinical outcomes in HCM patients and therefore its quantification has gained great importance. Endomyocardial biopsy, the gold standard invasive method, is used to assess interstitial collagen accumulation. CMR is emerging as a gold standard among the noninvasive imaging modalities to visualize the extracellular compartment of myocardium and quantify myocardial fibrosis. In a study of 29 patients by Moravsky et al. [13], the significant correlation was seen between quantitative assessment of myocardial fibrosis by histological methods and late gadolinium enhancement (LGE). The gadolinium contrast distributes preferentially to the extracellular compartment of the myocardium because it is small enough to pass through the capillary wall yet large enough to preclude its entry into the cell membrane (Fig. 3) [13]. The role of LGE on CMR for the prediction of major adverse outcomes such as SCD, arrhythmias, systolic and diastolic HF may be supported by some emerging data [14-16].

\section{Late gadolinium enhancement and SCD}

The performance of conventional risk factors for risk stratification of SCD in HCM is known

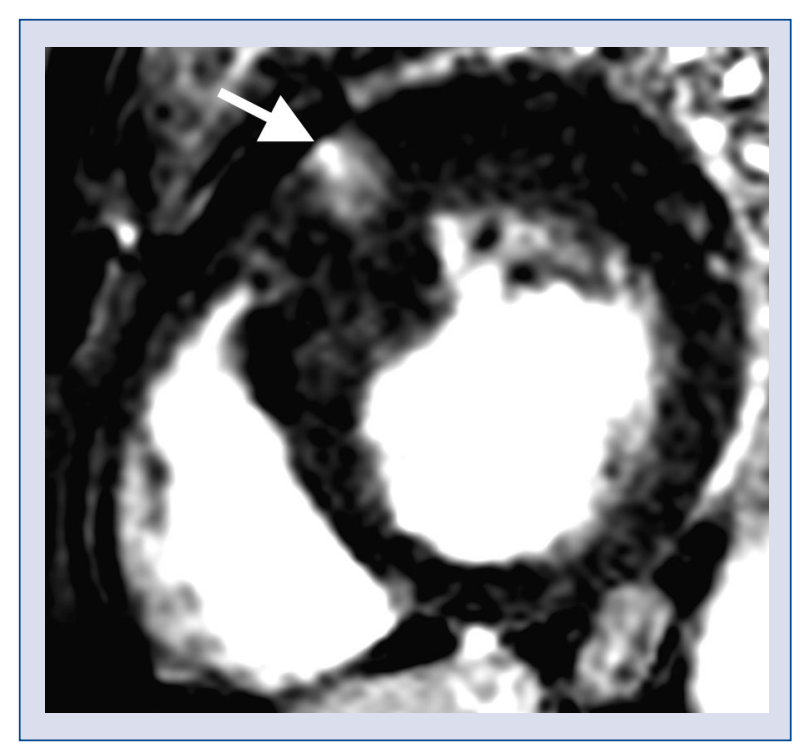

Figure 3. Short axis view on cardiac magnetic resonance with late gadolinium enhancement demonstrating patchy enhancement especially prominent in the anterior right ventricle insertion point (white arrow).

to be suboptimal. Hence, it can be challenging to accurately identify risk factors which can improve the patient selection for prophylactic ICD therapy [17]. Contrast-enhanced CMR has emerged as an excellent tool for improving risk stratification of HCM patients. Although the data are still limited in this regard, there are several studies which have highlighted that the presence and extent of LGE can improve risk stratification of HCM patients $[14,18-20]$. The relevant studies are summarized in Tables 1-4.

The presence and extent of LGE as a marker of myocardial fibrosis is a risk factor for SCD in HCM patients. Moon et al. [18] showed that greater area of LGE $(p<0.001)$ and the presence of two or more risk factors for sudden death $(\mathrm{p}<0.02)$ are directly proportional to disease progression in $\mathrm{HCM}$ patients. Progressive disease was defined as a decrease in maximal LV wall thickness by $\geq 5 \mathrm{~mm}$ and an increase in LV end-systolic dimension by $\geq 5 \mathrm{~mm}$ during 5 or more years of serial follow-up with an echocardiogram. In a recent study, Ismail et al. [21] have shown that even small increments in fibrosis, such as an increase in LGE by $5 \%$, significantly increase the risk of SCD. Another recent study by Chan et al. [22] virtually establishes that the extent of LGE is a strong predictor of SCD events. When compared with conventional risk factors, the extent of LGE was by far the best predictor of SCD events. When used in combination 


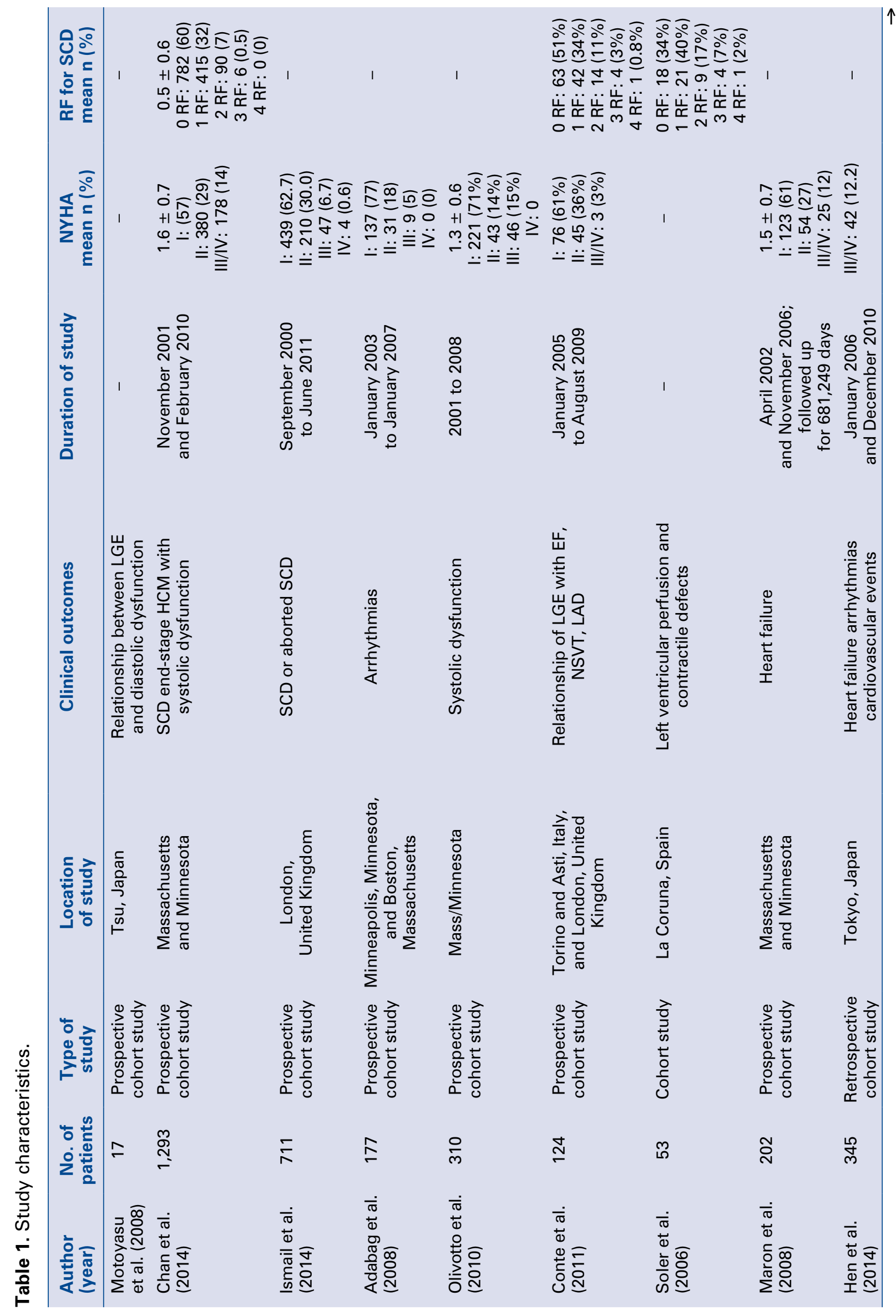




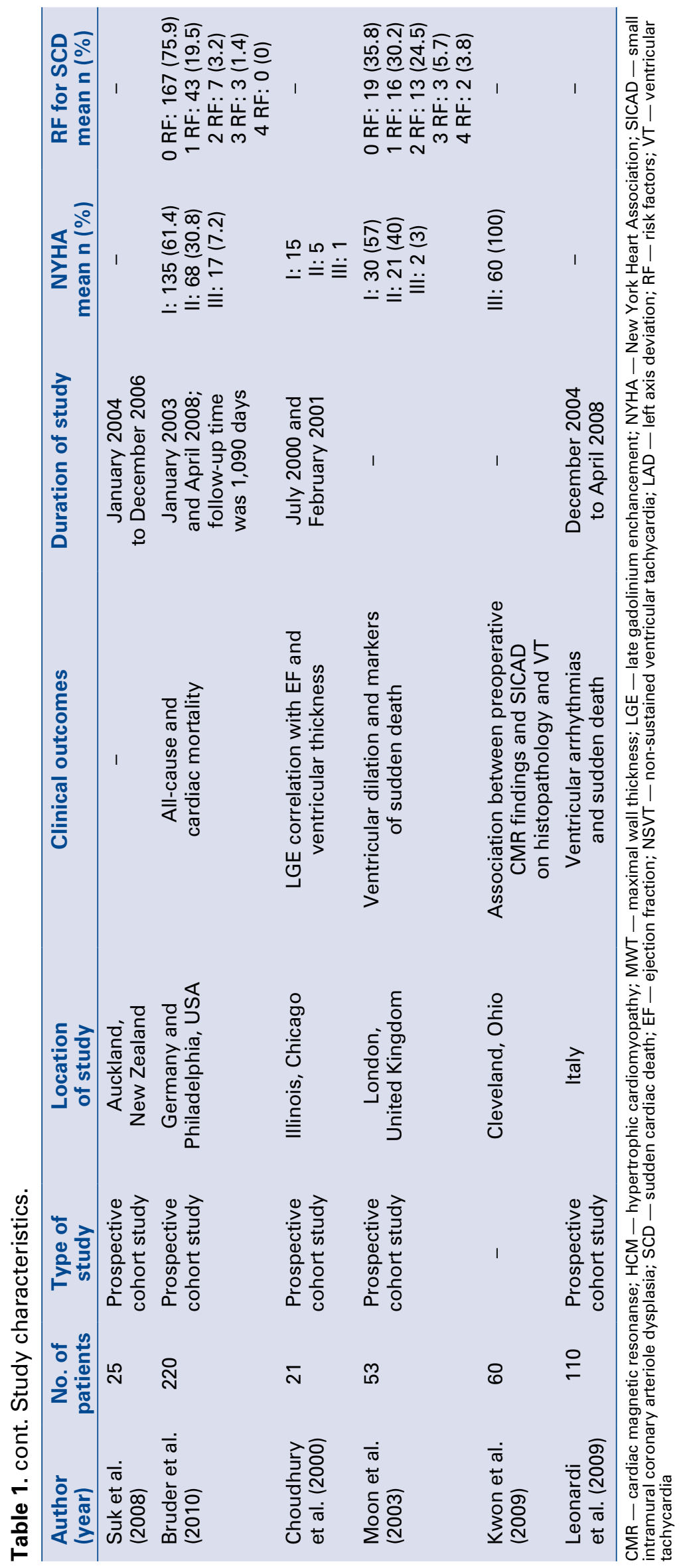


with other established predictors, the percentage of LGE significantly improved the prediction of SCD events. The results showed that increased LGE by $10 \%$ increases the risk of SCD events by $40 \%$ (HR: $1.46 / 10 \%$ increase in LGE, $\mathrm{p}=0.002$ ). The incidence of SCD per 1,000 person years was directly proportional to the percentage extent of LGE: 10 per 1,000 person years with $\mathrm{LGE} \leq 10 \%$, 18 per 1,000 person years with $11-19 \%$, and 24 per 1,000 person years with $\geq 20 \%$ ( $\mathrm{p}=0.001$ for trend [22]. Hen et al. [23] found a higher rate of cardiovascular events including SCD in the Japanese population with HCM who had LGE as compared to those without LGE (annual events rate, $6.2 \%$ per year vs. $0.6 \%$ per year, $\mathrm{p}=0.003$ ).

It is worthwhile to emphasize that the role of CMR extends even to those genotypically positive patients who have not yet developed the clinical signs or symptoms of disease. The presence of LGE in genotype (+)/phenotype(-) individuals can indicate structural abnormalities in non-hypertrophied muscle [24]. However, there is no established consensus regarding the definitive diagnostic and prognostic role of delayed enhancement for adverse disease outcomes like SCD and arrhythmias in such a setting.

\section{Late gadolinium enhancement and arrhythmias}

Myocardial fibrosis serves as an arrhythmogenic substrate for dangerous ventricular arrhythmias. The studies showing the relationship between LGE and arrhythmias are summarized in Tables 1-4. Adabag et al. [15] reported that patients with LGE had a 7-fold increased risk of nonsustained ventricular tachycardia as compared to those without it (relative risk: 7.3, 95\% CI 2.6-20.4, $\mathrm{p}<0.0001)$. Hen et al. [23] found a significantly higher percentage of arrhythmias in patients with LGE compared to patients without LGE in a Japanese cohort of HCM patients (annual events rate, $2.5 \%$ per year vs. $0 \%$ per year, $\mathrm{p}=0.037$ ). Suk et al. [25] demonstrated that the risk of ventricular arrhythmias could be indirectly predicted using LV mass. The size of myocardial scar and hence the risk of arrhythmias was found to be correlated with LV mass $\left(r^{2}=0.74\right)$ and LV wall thickness $\left(r^{2}=\right.$ $=0.42$ ). A myocardial scar mass of more than $7 \mathrm{~g}$ on LGE predicted the risk of developing ventricular tachycardia with $75 \%$ sensitivity and $82 \%$ specificity. Kwon et al. [16] independently confirmed an increased frequency of arrhythmias using Holter monitoring. It was reported that a greater per- centage of patients with LGE had documented arrhythmias compared to those without LGE (27\% vs. $5 \%, \mathrm{p}=0.03$ ). Leonardi et al. [26] devised a scoring system for quantitative assessment of LGE in HCM known as Delayed contrast enhancement score. Points were given to each segment based on the percentage of LGE (i.e. $0-$ absence of LGE; $1-<25 \%$ LGE; $2-25-50 \%$ of LGE; and $3->50 \%$ of LGE). The total score comprised of individual scores from all segments. Patients were divided into two main groups: group $\mathrm{R}$ (risk) with risk factors and group LR (low risk) without any risk factors for SCD. Group R was further divided into high-risk (HR) and intermediate risk (IR) subpopulations. The HR subgroup included patients with prior history of cardiac arrest or sustained ventricular tachycardia and the presence of at least two other risk factors, whereas the IR subgroup included patients with only one risk factor. In the analysis conducted by Leonardi et al. [26], using delayed contrast enhancement score, LGE was also the only independent predictor of ventricular arrhythmias (OR: 1.073, 1.023-1.125, p = 0.004) in the multivariable analysis.

\section{Late gadolinium enhancement and systolic dysfunction}

Systolic impairment in HCM results from replacement fibrosis, which occurs as a consequence of microvascular ischemia and myocyte death [27]. Sarcomere mutations cause metabolically inefficient contractions and myocyte energy depletion which can further contribute to systolic dysfunction by triggering apoptosis and collagen deposition $[28,29]$. Therefore, the extent of LGE can predict the risk of systolic dysfunction. Various studies have analyzed the relationship between LGE and systolic dysfunction, and they are summarized in Tables 1-4.

The systolic dysfunction can occur at any point during HCM. Chan et al. [22] described the risk of development of systolic dysfunction in end-stage HCM patients. Every $10 \%$ increase in LGE resulted in a significant decrease in systolic function (HR: 1.80, 1.40-2.40, p < 0.03). In the prospective cohort study conducted by Ismail et al. [21] in HCM patients with LV dysfunction, there was evidence of fibrosis on CMR. Olivotto et al. [30] demonstrated that LGE predicts adverse LV remodeling in HCM patients and it is negatively correlated to EF. A subgroup of $\mathrm{HCM}$ patients (NYHA I or II) with EF 50-65\% are in the transition phase towards advanced LV remodeling and 
Table 2. Patient demographics.

\begin{tabular}{|c|c|c|c|c|}
\hline Author (year) & $\begin{array}{l}\text { Age (mean } \pm \\
\text { standard devia- } \\
\text { tion) [years] }\end{array}$ & $\begin{array}{l}\text { Male } \\
\text { n (\%) }\end{array}$ & $\begin{array}{l}\text { Arrhythmias } \\
\qquad \mathrm{n}(\%)\end{array}$ & $\begin{array}{l}\text { Mortality during follow-up } \\
n(\%)\end{array}$ \\
\hline $\begin{array}{l}\text { Motoyasu et al. } \\
\text { (2008) }\end{array}$ & $57.7 \pm 9.8$ & $13(76)$ & - & - \\
\hline $\begin{array}{l}\text { Chan et al. } \\
\text { (2014) }\end{array}$ & $46 \pm 17$ & $85(63)$ & $\begin{array}{l}\text { NSVT } 204(20) \\
\text { AF } 159(12)\end{array}$ & $\begin{array}{c}\text { HCM related SD } 14(1.0) \\
\text { Aborted arrest } 6(0.5) \\
\text { ICD discharge (VT/VF) 17 (1.3) } \\
\text { HF death } 6(0.5) \\
\text { HT } 9(0.7) \\
\text { End-stage HCM } 87(7) \\
\text { NYHA class III/IV } 99(9) \\
\text { NCD } 21(1.6)\end{array}$ \\
\hline $\begin{array}{l}\text { Ismail et al. } \\
\text { (2014) }\end{array}$ & 56.3 (median) & $498(70)$ & $\begin{array}{c}\text { AF } 22(3.2) \\
\text { NSVT } 38(5.4)\end{array}$ & - \\
\hline $\begin{array}{l}\text { Adabag et al. } \\
(2008)\end{array}$ & $41 \pm 16$ & $129(73)$ & - & \\
\hline $\begin{array}{l}\text { Olivotto et al. } \\
\text { (2010) }\end{array}$ & $42 \pm 17$ & $218(70)$ & & - \\
\hline $\begin{array}{l}\text { Conte et al. } \\
\text { (2011) }\end{array}$ & $53 \pm 17$ & $86(69)$ & $\begin{array}{l}\text { NSVT } 20 / 182, p=0.001 \\
\text { AF } 17 / 182, p=0.19\end{array}$ & - \\
\hline Soler et al. (2006) & $49.55 \pm 14.24$ & $33(62)$ & - & - \\
\hline Maron et al. (2008) & $42 \pm 17$ & $144(71)$ & - & - \\
\hline Hen et al. (2014) & $59 \pm 17$ & $214(62)$ & AF 65 (18.8) & - \\
\hline Suk et al. (2008) & $54 \pm 8$ & $17(68)$ & - & - \\
\hline $\begin{array}{l}\text { Bruder et al. } \\
(2010)\end{array}$ & 58 & $149(61.4)$ & - & $\begin{array}{l}\text { All-cause mortality } 22(10.0) \\
\text { Cardiac mortality } 16(7.2)\end{array}$ \\
\hline $\begin{array}{l}\text { Choudhury et al. } \\
(2000)\end{array}$ & 39 (median age) & $12(57)$ & - & None \\
\hline $\begin{array}{l}\text { Moon et al. } \\
(2003)\end{array}$ & $\begin{array}{l}47 \pm 16 \\
\text { (range } 15-73 \text { ) }\end{array}$ & $37(70)$ & $\begin{array}{c}\text { NSVT on Holter } \\
\text { monitor } 11(21 \%) \\
\text { Documented sustained } \\
\text { VT/VF } 3(6 \%)\end{array}$ & \\
\hline Kwon et al. (2009) & $51 \pm 14$ & $37(62)$ & & \\
\hline $\begin{array}{l}\text { Leonardi et al. } \\
\text { (2009) }\end{array}$ & $42 \pm 15$ & $82(76)$ & & \\
\hline
\end{tabular}

AF — atrial fibrillation; HCM — hypertrophic cardiomyopathy; ICD — implantable cardioverter defibrillator; HF — heart failure; HT — heart transplantation; NCD - non cardiac death; NSVT — non-sustained ventricular tachycardia; NYHA — New York Heart Association; SD - sudden death; VT/VF - ventricular tachycardia/ventricular fibrillation

systolic failure. Therefore, regular clinical surveillance and prophylactic therapies have fundamental importance in the management of these patients. In another study by Conte et al. [31], patients were divided into three groups according to the number of segments positive for LGE (first group, $0.3 \pm$ \pm 0.4 ; second group, $2.2 \pm 0.4$; third group, $5.2 \pm$ \pm 1.9 segments). The percentage of patients with $\mathrm{EF}<50 \%$ as assessed by CMR, in these groups was: group $1(4 \%)$, group $2(4 \%)$, group $3(17 \%), p=$ $=0.02$. Thus, it was suggested that patients who had a large extent of LGE on CMR had higher risk of systolic dysfunction. While analyzing the rela- tionship between delayed enhancement and contractile functions in $53 \mathrm{HCM}$ patients with CMR, Soler et al. [32] showed that significant correlations existed between delayed enhancement (DE) and hypokinetic segments $(\mathrm{r}=0.3, \mathrm{p}<0.05)$. Maron et al. [33] demonstrated in a large cohort of HCM patients the association of $\mathrm{LGE}$ and presence of $\mathrm{HF}$ symptoms $(p=0.05)$ along with LV systolic dysfunction ( $p=0.001)$. Hen et al. [23] stratified patients into three groups according to the LGE score (no LGE, 0; mild LGE, 1-3; marked LGE, > 4). LGE was converted to scores on a scale of 0 to 17 , as the sum of the segments in the 17 -segment 


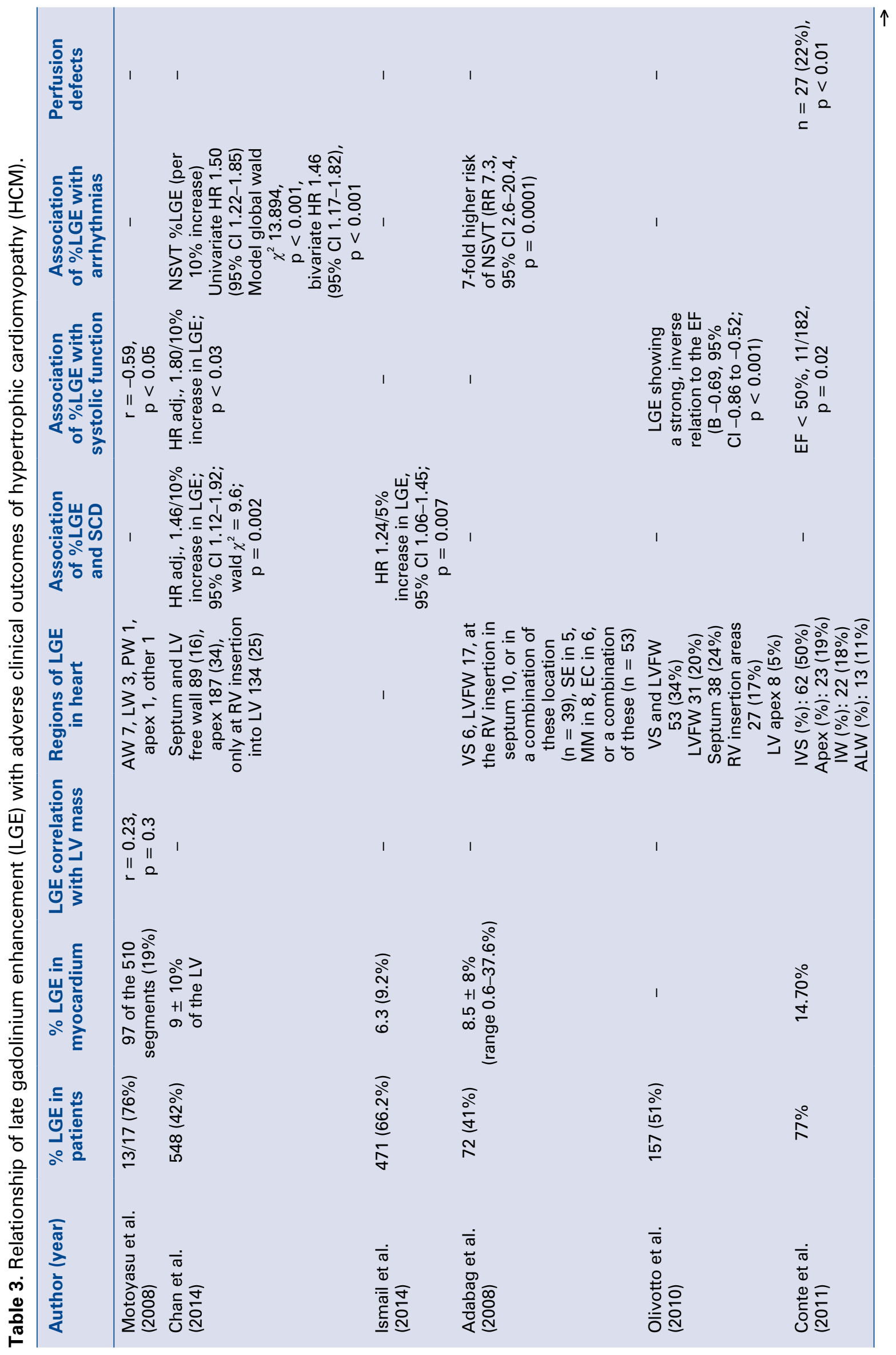




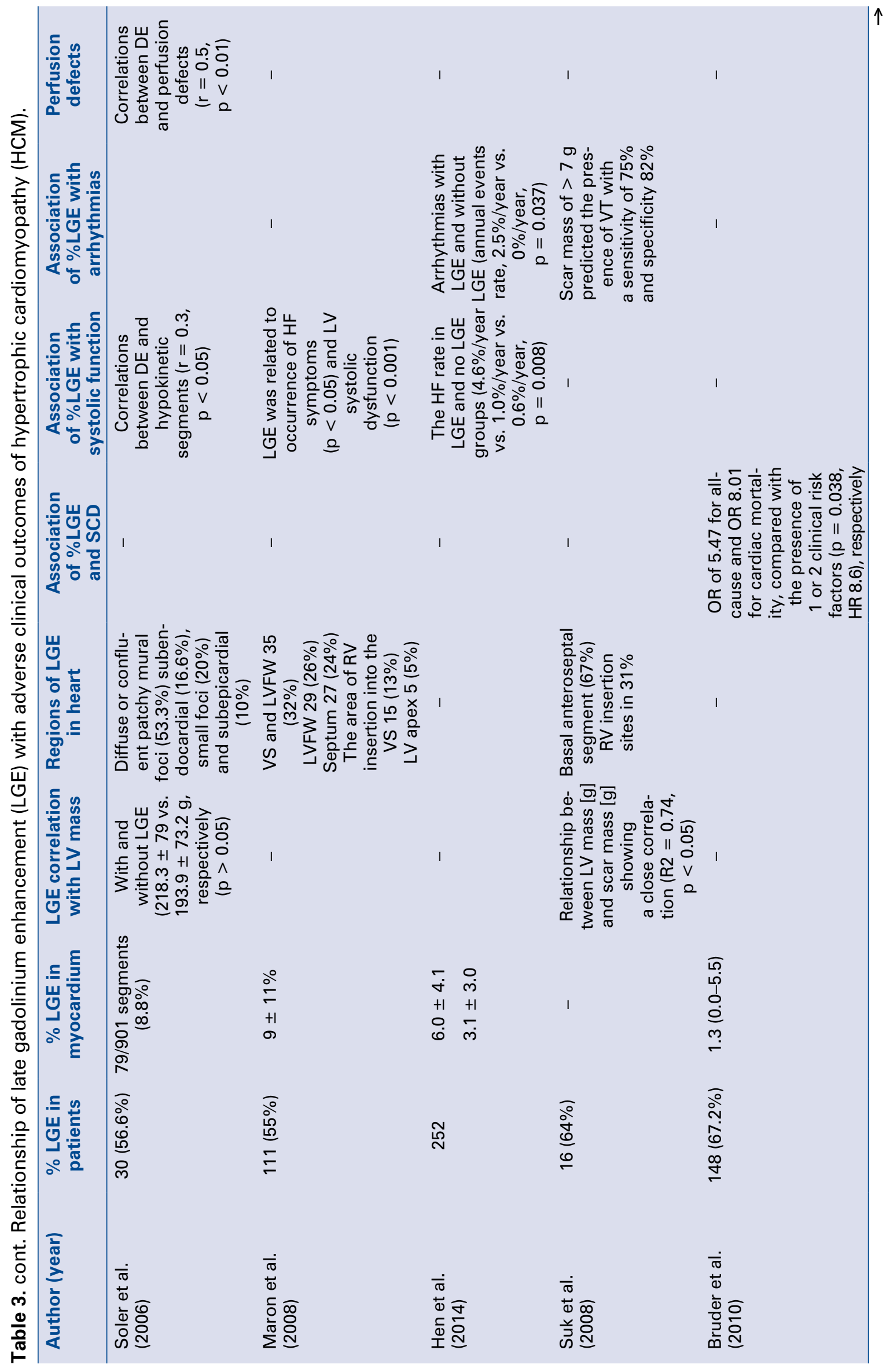




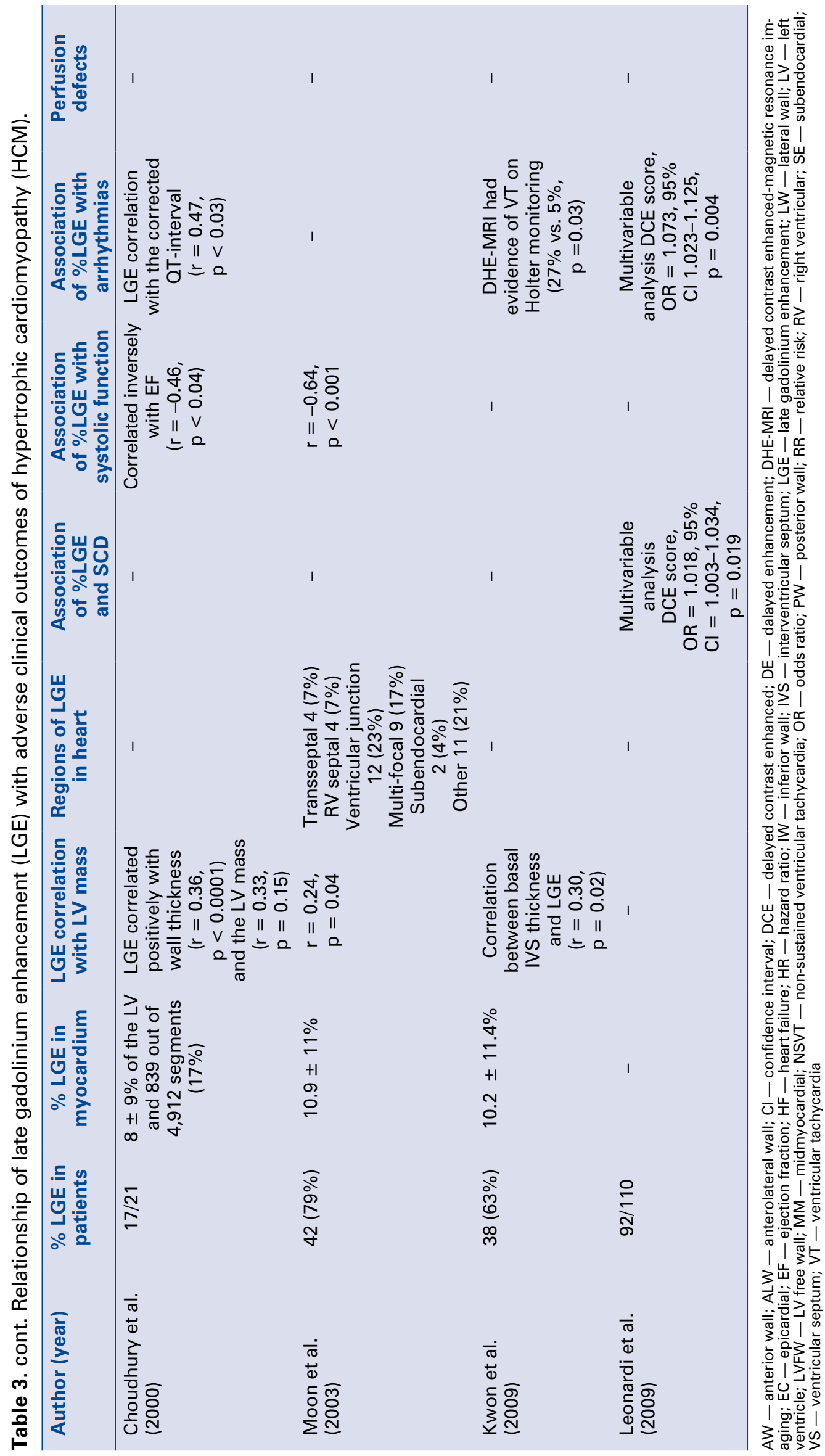




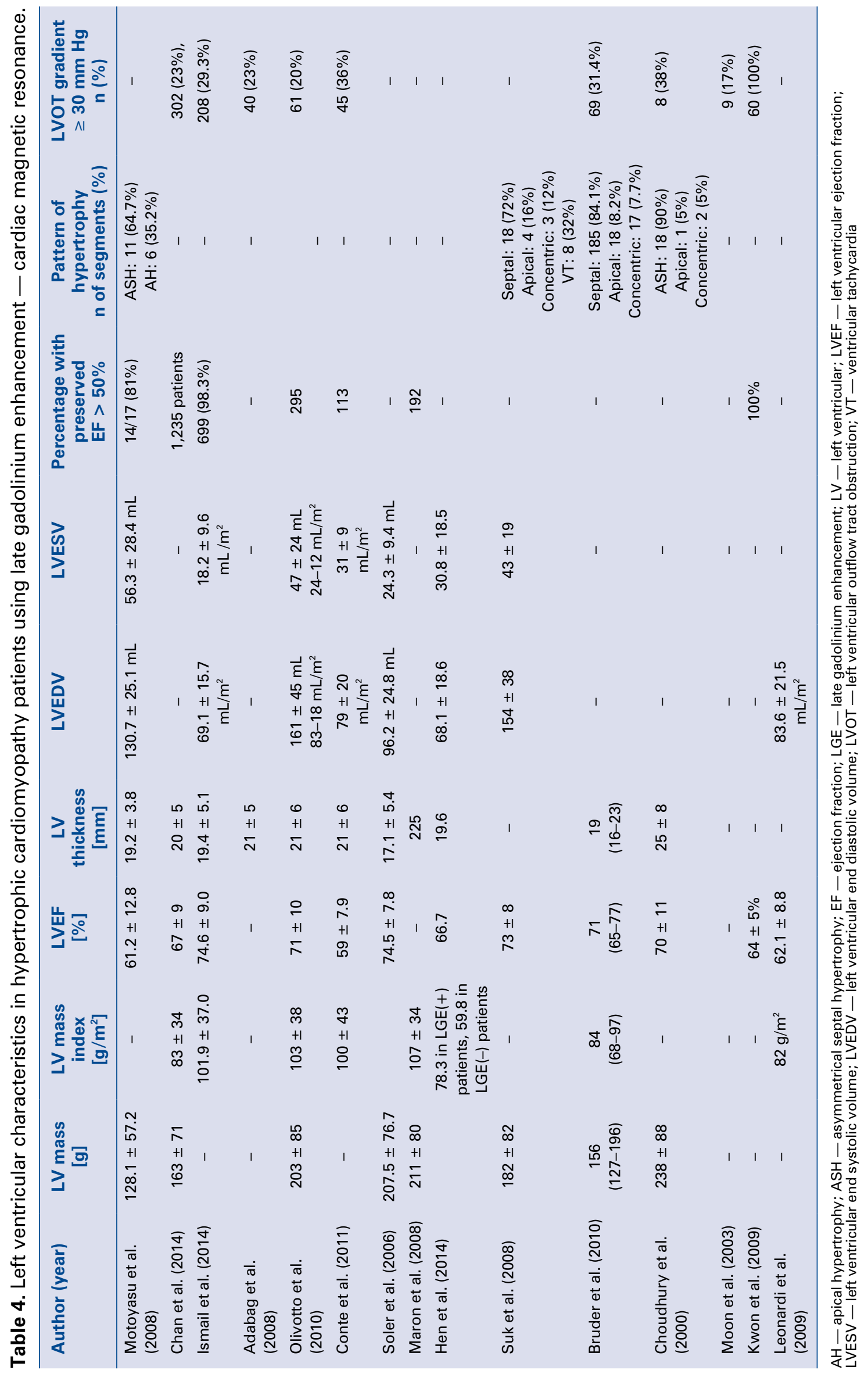




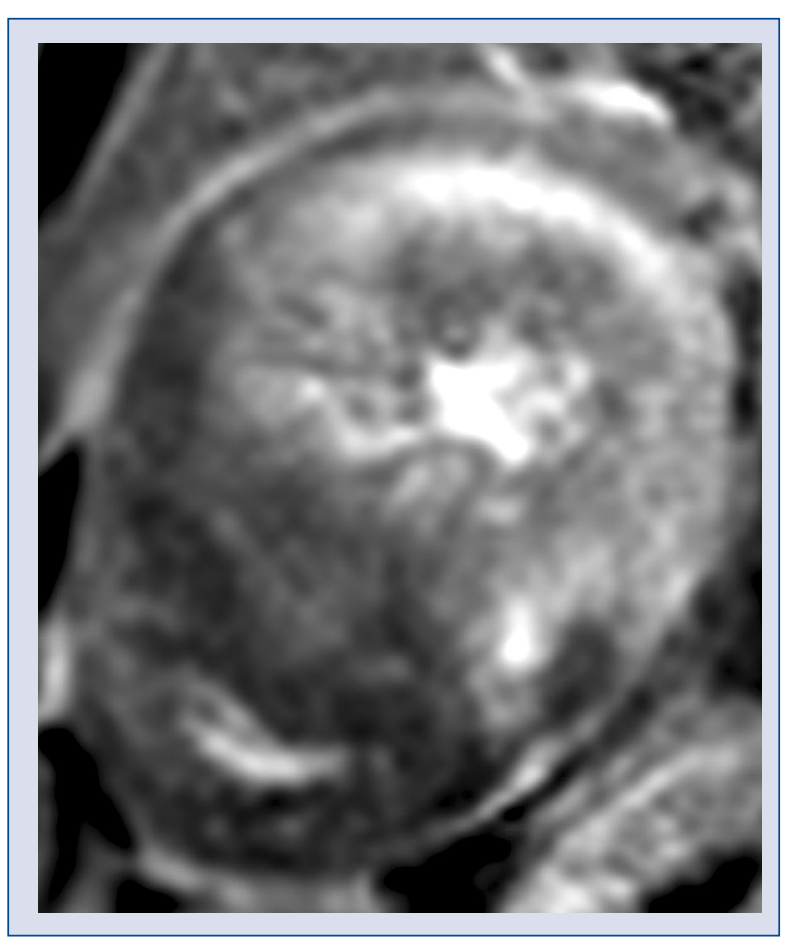

Figure 4. Short axis view on cardiac magnetic resonance with late gadolinium enhancement demonstrating diffuse enhancement of the left ventricular myocardium.

model showing LGE. The HF rate was higher in marked LGE vs. mild LGE groups (4.6\% per year vs. $1.0 \%$ per year vs. $0.6 \%$ per year, $p=0.008)$. According to Choudhury et al. [20], the extent of LGE modestly correlates with EF $(r=-0.64, \mathrm{p}<0.001)$.

\section{Late gadolinium enhancement and diastolic dysfunction}

It is well established that diastolic dysfunction precedes systolic dysfunction in the course of HCM. Motoyasu et al. [34] showed that strong negative correlation exists between LGE and diastolic dysfunction evidenced by decreased peak filling rate $(r=-0.86, p<0.01)$.

\section{The role of T1 mapping for detection of diffuse myocardial fibrosis in HCM}

Endomyocardial biopsy, the gold standard for myocardial fibrosis, is an invasive procedure associated with morbidity, sampling error and sampling limitations [35]. Therefore, myocardial fibrosis is preferably determined using LGE. Although LGE remains an excellent tool for detecting focal myocardial fibrosis, detection of diffuse fibrosis (Fig. 4) remains challenging since the normal myocardium is used as a reference to highlight patchy areas of focal myocardial fibrosis [36]. Extracellular volume (ECV) quantification by T1 mapping with CMR has overcome this limitation of LGE for detecting diffuse fibrosis in HCM. ECV measures T1 relaxation time, which is based on the molecular environment of water molecules. Every tissue has its characteristic T1 relaxation time, but depending on the pathophysiological state, it can vary within the same tissue. ECV can be measured with and without contrast and has shown promising results for the detection of myocardial fibrosis. Myocardial T1 measurement without the use of gadolinium contrast agents is known as native $\mathrm{T} 1$, which shows cellular and extracellular portions of the myocardium. T1 measurement at a point of time after giving a bolus of contrast is known as post contrast $\mathrm{T} 1$ and can reveal myocardial properties mostly at the cellular level [37]. Post-contrast T1 values are shorter than pre-contrast or native $\mathrm{T} 1$ images due to a small amount of gadolinium contrast being left in the myocardial interstitium during slow renal washout. Renal clearance of gadolinium, hematocrit, contrast dosage and time measurements are potential confounding variables for post contrast T1 [38]. T1 mapping is done systematically in which pixel intensity is directly proportional to T1 relaxation time of the corresponding myocardial voxel. This leads to signal quantification of each myocardial voxel on a standardized scale with high resolution. ECV measurements also hold strong histological validation and closely correlate with collagen volume fraction [39]. ECV quantification by T1 mapping has significant prognostic implications. Wong et al. [40] have demonstrated an association between myocardial extracellular expansion and adverse clinical outcomes using ECV. T1 mapping techniques have another advantage of detecting diffuse fibrosis at early stages of the disease. Compared to LGE images, T1 mapping CMR techniques allow us to eliminate the influences of windowing and variations in signal enhancement by directly measuring the underlying $\mathrm{T} 1$ relaxation times. Therefore, it allows signal quantification (in ms) on a standardized scale of each myocardial voxel to characterize myocardial tissue.

\section{Conclusions}

Echocardiography is currently the most widely used imaging modality in HCM. Although echocardiography is an excellent non-invasive modality, there are shortcomings in the comprehensive 
evaluation and risk stratification of $\mathrm{HCM}$ patients if utilized exclusively. SCD is the most feared outcome of HCM. Myocardial fibrosis in HCM can initiate malignant cardiac arrhythmias and SCD even in the absence of conventional risk factors. The extent of LGE on CMR is emerging as a potential risk factor for SCD in HCM patients. CMR has the additional capability of quantifying myocardial fibrosis using novel techniques such as $\mathrm{T} 1$ mapping. CMR has incremental value and is complementary to echocardiography in the comprehensive evaluation of patients with HCM.

\section{Conflict of interest: None declared}

\section{References}

1. Bos JM, Towbin JA, Ackerman MJ. Diagnostic, prognostic, and therapeutic implications of genetic testing for hypertrophic cardiomyopathy. J Am Coll Cardiol, 2009; 54: 201-211. doi: 10.1016/j. jacc.2009.02.075.

2. Keren A, Syrris P, McKenna WJ. Hypertrophic cardiomyopathy: The genetic determinants of clinical disease expression. Nat Clin Pract Cardiovasc Med, 2008; 5: 158-168. doi: 10.1038/ ncpcardio1110.

3. Maron BJ, Maron MS. Hypertrophic cardiomyopathy. Lancet, 2013; 381: 242-255. doi: 10.1016/s0140-6736(12)60397-3.

4. Maron BJ, Casey SA, Hauser RG, Aeppli DM. Clinical course of hypertrophic cardiomyopathy with survival to advanced age. J Am Coll Cardiol, 2003; 42: 882-888.

5. Elliott PM, Poloniecki J, Dickie S et al. Sudden death in hypertrophic cardiomyopathy: Identification of high risk patients. J Am Coll Cardiol, 2000; 36: 2212-2218.

6. Gersh BJ, Maron BJ, Bonow RO et al. 2011 ACCF/AHA guideline for the diagnosis and treatment of hypertrophic cardiomyopathy: Executive summary: A report of the American College of Cardiology Foundation/American Heart Association Task Force on Practice Guidelines. Circulation, 2011; 124: 2761-2796. doi: 10.1161/CIR.0b013e318223e230.

7. Watkins H, Ashrafian H, Redwood C. Inherited cardiomyopathies. N Engl J Med, 2011; 364: 1643-1656. doi: 10.1056/NEJMra0902923.

8. Maron MS, Maron BJ, Harrigan C et al. Hypertrophic cardiomyopathy phenotype revisited after 50 years with cardiovascular magnetic resonance. J Am Coll Cardiol, 2009; 54: 220-228. doi: 10.1016/j.jacc.2009.05.006.

9. Maron BJ, Pelliccia A, Spataro A, Granata M. Reduction in left ventricular wall thickness after deconditioning in highly trained Olympic athletes. Br Heart J, 1993; 69: 125-128.

10. Pelliccia A, Maron BJ, De Luca R, Di Paolo FM, Spataro A, Culasso F. Remodeling of left ventricular hypertrophy in elite athletes after long-term deconditioning. Circulation, 2002; 105: 944-949.

11. Schaper J, Speiser B. The extracellular matrix in the failing human heart. Basic Res Cardiol, 1992; 87 (suppl. 1): 303-309.

12. Khan R, Sheppard R. Fibrosis in heart disease: Understanding the role of transforming growth factor-beta in cardiomyopathy, valvular disease and arrhythmia. Immunology, 2006; 118: 10-24. doi: 10.1111/j.1365-2567.2006.02336.x.

13. Moravsky G, Ofek E, Rakowski $\mathrm{H}$ et al. Myocardial fibrosis in hypertrophic cardiomyopathy: Accurate reflection of histopatho- logical findings by CMR. J Am Coll Cardiol Cardiovasc Imaging, 2013; 6: 587-596. doi: 10.1016/j.jcmg.2012.09.018.

14. Bruder O, Wagner A, Jensen CJ et al. Myocardial scar visualized by cardiovascular magnetic resonance imaging predicts major adverse events in patients with hypertrophic cardiomyopathy. J Am Coll Cardiol, 2010; 56: 875-887. doi: 10.1016/j.jacc.2010.05.007.

15. Adabag AS, Maron BJ, Appelbaum E et al. Occurrence and frequency of arrhythmias in hypertrophic cardiomyopathy in relation to delayed enhancement on cardiovascular magnetic resonance. J Am Coll Cardiol, 2008; 51: 1369-1374. doi: 10.1016/j. jacc.2007.11.071.

16. Kwon DH, Setser RM, Popovic ZB et al. Association of myocardial fibrosis, electrocardiography and ventricular tachyarrhythmia in hypertrophic cardiomyopathy: A delayed contrast enhanced MRI study. Int J Cardiovasc Imaging, 2008; 24: 617-625. doi: 10.1007/s10554-008-9292-6.

17. Maron BJ. Contemporary insights and strategies for risk stratification and prevention of sudden death in hypertrophic cardiomyopathy. Circulation, 2010; 121: 445-456. doi: 10.1161/circulationaha.109.878579.

18. Moon JC, McKenna WJ, McCrohon JA, Elliott PM, Smith GC, Pennell DJ. Toward clinical risk assessment in hypertrophic cardiomyopathy with gadolinium cardiovascular magnetic resonance. J Am Coll Cardiol, 2003; 41: 1561-1567.

19. Green JJ, Berger JS, Kramer CM, Salerno M. Prognostic value of late gadolinium enhancement in clinical outcomes for hypertrophic cardiomyopathy. J Am Coll Cardiol Cardiovasc Imaging, 2012; 5: 370-377. doi: 10.1016/j.jcmg.2011.11.021.

20. Choudhury L, Mahrholdt H, Wagner A et al. Myocardial scarring in asymptomatic or mildly symptomatic patients with hypertrophic cardiomyopathy. J Am Coll Cardiol, 2002; 40: 2156-2164.

21. Ismail TF, Jabbour A, Gulati A et al. Role of late gadolinium enhancement cardiovascular magnetic resonance in the risk stratification of hypertrophic cardiomyopathy. Heart, 2014; 100: 1851-1858. doi: 10.1136/heartjnl-2013-305471.

22. Chan RH, Maron BJ, Olivotto I et al. Prognostic value of quantitative contrast-enhanced cardiovascular magnetic resonance for the evaluation of sudden death risk in patients with hypertrophic cardiomyopathy. Circulation, 2014; 130: 484-495. doi: 10.1161/ circulationaha.113.007094.

23. Hen $\mathrm{Y}$, Iguchi N, Utanohara $\mathrm{Y}$ et al. Prognostic value of late gadolinium enhancement on cardiac magnetic resonance imaging in Japanese hypertrophic cardiomyopathy patients. Circ J, 2014; 78: 929-937.

24. Rowin EJ, Maron MS, Lesser JR, Maron BJ. CMR with late gadolinium enhancement in genotype positive-phenotype negative hypertrophic cardiomyopathy. J Am Coll Cardiol Cardiovasc Imaging, 2012; 5: 119-122. doi: 10.1016/j.jcmg.2011.08.020.

25. Suk T, Edwards C, Hart H, Christiansen JP. Myocardial scar detected by contrast-enhanced cardiac magnetic resonance imaging is associated with ventricular tachycardia in hypertrophic cardiomyopathy patients. Heart Lung Circ, 2008; 17: 370-374. doi: 10.1016/j.hlc.2008.03.080.

26. Leonardi S, Raineri C, De Ferrari GM et al. Usefulness of cardiac magnetic resonance in assessing the risk of ventricular arrhythmias and sudden death in patients with hypertrophic cardiomyopathy. Eur Heart J, 2009; 30: 2003-2010. doi: 10.1093/ eurheartj/ehp152.

27. Harris KM, Spirito P, Maron MS et al. Prevalence, clinical profile, and significance of left ventricular remodeling in the end-stage phase of hypertrophic cardiomyopathy. Circulation, 2006; 114: 216-225. doi: 10.1161/circulationaha.105.583500.

28. Ashrafian H, Redwood C, Blair E, Watkins H. Hypertrophic cardiomyopathy: A paradigm for myocardial energy depletion. Trends Genet, 2003; 19: 263-268. doi: 10.1016/s0168-9525(03)00081-7. 
29. Girolami F, Ho CY, Semsarian C et al. Clinical features and outcome of hypertrophic cardiomyopathy associated with triple sarcomere protein gene mutations. J Am Coll Cardiol, 2010; 55: 1444-1453. doi: 10.1016/j.jacc.2009.11.062.

30. Olivotto I, Maron BJ, Appelbaum E et al. Spectrum and clinical significance of systolic function and myocardial fibrosis assessed by cardiovascular magnetic resonance in hypertrophic cardiomyopathy. Am J Cardiol, 2010; 106: 261-267. doi: 10.1016/j. amjcard.2010.03.020.

31. Conte MR, Bongioanni S, Chiribiri A et al. Late gadolinium enhancement on cardiac magnetic resonance and phenotypic expression in hypertrophic cardiomyopathy. Am Heart J, 2011; 161: 1073-1077. doi: 10.1016/j.ahj.2011.03.022.

32. Soler R, Rodriguez E, Monserrat L, Mendez C, Martinez C. Magnetic resonance imaging of delayed enhancement in hypertrophic cardiomyopathy: Relationship with left ventricular perfusion and contractile function. J Comput Assist Tomogr, 2006; 30: 412-420.

33. Maron MS, Appelbaum E, Harrigan CJ et al. Clinical profile and significance of delayed enhancement in hypertrophic cardiomyopathy. Circ Heart Fail, 2008; 1: 184-191. doi: 10.1161/circheartfailure.108.768119.

34. Motoyasu M, Kurita T, Onishi $\mathrm{K}$ et al. Correlation between late gadolinium enhancement and diastolic function in hypertrophic cardiomyopathy assessed by magnetic resonance imaging. Circ J, 2008; 72: 378-383.

35. Schalla S, Bekkers SC, Dennert R et al. Replacement and reactive myocardial fibrosis in idiopathic dilated cardiomyopathy:
Comparison of magnetic resonance imaging with right ventricular biopsy. Eur J Heart Fail, 2010; 12: 227-231. doi: 10.1093/ /eurjhf/hfq004.

36. Mongeon FP, Jerosch-Herold M, Coelho-Filho OR, Blankstein R, Falk RH, Kwong RY. Quantification of extracellular matrix expansion by CMR in infiltrative heart disease. J Am Coll Cardiol Cardiovasc Imaging, 2012; 5: 897-907. doi: 10.1016/j. jcmg.2012.04.006.

37. Moon JC, Messroghli DR, Kellman P et al. Myocardial T1 mapping and extracellular volume quantification: A Society for Cardiovascular Magnetic Resonance (SCMR) and CMR Working Group of the European Society of Cardiology consensus statement. J Cardiovasc Magn Reson, 2013; 15: 92. doi: 10.1186/1532429x-15-92.

38. Iles L, Pfluger H, Phrommintikul A et al. Evaluation of diffuse myocardial fibrosis in heart failure with cardiac magnetic resonance contrast-enhanced T1 mapping. J Am Coll Cardiol, 2008; 52: 1574-1580. doi: 10.1016/j.jacc.2008.06.049.

39. Miller CA, Naish JH, Bishop P et al. Comprehensive validation of cardiovascular magnetic resonance techniques for the assessment of myocardial extracellular volume. Circ Cardiovasc Imaging, 2013; 6: 373-383. doi: 10.1161/circimaging.112.000192.

40. Wong TC, Piehler KM, Kang IA et al. Myocardial extracellular volume fraction quantified by cardiovascular magnetic resonance is increased in diabetes and associated with mortality and incident heart failure admission. Eur Heart J, 2014; 35: 657-664. doi: 10.1093/eurheartj/eht193. 